\title{
The "Sensitive" Markovian Queueing System and Its Application for a Call Center Problem
}

\author{
Odysseas Kanavetas • Barış Balcıog̃lu
}

Received: date / Accepted: date

\begin{abstract}
In this paper, we study the $M_{n} / M_{n} / c / K+M_{n}$ queueing system where customers arrive according to a Poisson process with state-dependent rates. Moreover, the rates of the exponential service times and times to abandonment of the queued customers can also change whenever the system size changes. This implies that a customer may experience different service rates throughout the time she is being served. Similarly, a queued customer can change her patience time limits while waiting in the queue. Thus, we refer to the analyzed system as the "sensitive" Markovian queue. We conduct an exact analysis of this system and obtain its steady-state performance measures. The steady-state system size distribution yields itself via a birth-death process. The times spent in the queue by an arbitrary or an eventually served customer are represented as the times until absorption in two continuous-time Markov chains and follow Phase-type distributions with which the queueing time distributions and moments are obtained. Then, we demonstrate how the $M_{n} / M_{n} / c / K+M_{n}$ queue can be employed to approximately yet accurately estimate the performance measures of the $M_{n} / G I / c / K+G I$ type call center.
\end{abstract}

Keywords The $M_{n} / M_{n} / c / K+M_{n}$ queue $\cdot$ The $M_{n} / G I / c / K+G I$ queue $\cdot$ Impatient customers · Phase-type distribution · Call centers

\section{Introduction}

With the advanced technology that is able to continuously monitor the changes in the system and process the data collected, many delay systems, such as call

College of Engineering, Koç University

Sarıer, 34450 Istanbul, Turkey

E-mail: okanavetas@ku.edu.tr

Faculty of Engineering and Natural Sciences, Sabancı University

Orhanll-Tuzla, 34956 Istanbul, Turkey

E-mail: balcioglu@sabanciuniv.edu 
centers, healthcare facilities, sharing some sort of waiting time estimates have become indirectly observable queues from the customers' point of view. The number of other customers found upon arrival who wait to be served as well as the perceived service speed (via updated waiting time estimates provided by the service provider) are used by customers to decide whether to join the queue and to give up on waiting and leave the system. The changes in the amount of workload can also impact the service speed. When the number of customers waiting for service increases, taking the initiative the servers can speed up. On the other hand, paced up speed for long periods of time may fatigue the servers who may tactically slow down to induce some of the queued customers to abandon. To encompass all this dynamic behavior that the customers and servers can exhibit depending on the changes in the workload in the system, in this paper, we consider the $M_{n} / M_{n} / c / K+M_{n}$ queueing system. Since all the rates of the underlying exponential distributions for time between arrivals, service times, and times-to-abandonment can change with the change in the number of customers in the system, we refer to this queue as the "sensitive" Markovian queueing system. In this study, we obtain the frequently employed steady-state performance measures of this system with an exact method, focusing particularly on the derivation of the queueing time distributions and moments of the served and reneging customers. As a secondary motive, we also construct an $M_{n} / M_{n} / c / K+M_{n}$ queue based approximation to capture the statistics of the $M_{n} / G I / c / K+G I$ queue, used to model call centers where service and time-to-abandon distributions can be general.

In traditional visible queueing systems, such as in stores, depending on how she perceives the size of the workload to be processed before she can be served, a newly arriving customer may choose to stay or leave the system. This typical example is illustrative of why arrival rates can be state-dependent. More popularly, dynamic pricing schemes, with its growing rich literature since Naor (1969), implemented by service providers also control and vary the customer arrival rate. For example, when on-hand stock levels decrease, by increasing the price of the product, the demand rate is lowered. This, consequently, decreases the rate of production orders when high prices are posted, making the arrival process at the production facility also state-dependent (see the review by Elmaghraby and Keskinocak, 2003, for other examples). Thus, visualizing state-dependent Poisson processes to model the customer arrivals is common in queueing analysis. Providing delay information at call centers has also become common practice and its impact on the arrival of calls has attracted attention in the literature (e.g., Armony, Shimkin, and Whitt, 2009).

When customers are impatient, on the other hand, it is common to assume a single patience time distribution for all customers ignoring the fact that the direct or indirect information on the queue length at the moment of arrival may have an impact on patience limits. A single time-to-abandonment distribution can be a valid assumption if queues are completely unobservable by the customers, i.e., customers do not have any access to some sort of delay information (see Iravani and Balcıoglu, 2008, and the references therein). In call centers providing and updating delay information (Jouini et al., 2014), 
it is noted that reneging behavior may change with delay announcement as well (Jouini, Akşin, and Dallery, 2011). As considered by Armony, Plambeck, and Seshardi (2009), total abandonment rate can be expressed as a function of the number of customers in the system. Total abandonment rate can become state-dependent due to different patience limits a customer may set and update depending on her perception of the workload in the system. It is reasonable to assume that a customer with a long or short informed waiting time in front of her will set different patience limits. And after starting to wait in the queue, receiving updates on how the workload in front of her decreases she may also update her patience limit for waiting further for service.

One can find examples of state-dependent service rates in a wide range of settings as well: The regulation of transmission rates depending on the buffer content in packet-switched telecommunication networks, collected water volume dependent releases from dams, speed of traffic on highway depending on the traffic density, etc. For profit maximization, Ata and Shneorson (2006) suggest dynamically controlling the service rates along with the usage of dynamic pricing policies. Obviously service rate can be controlled more easily with automatic and computerized servers. When servers are human beings, initiatives taken on a personal level or wearing out due to the fatigue can affect the service rate. For state-dependent service rates depending on the workload, Tan and Netessine (2014) provide an example from a restaurant chain, Berry-Jaeker and Tucker (2016) and Batt and Terwiesch (2016) observe non-monotone relationship between workload and service rate in hospitals.

In this context, the most flexible Markovian queueing system to address the dynamic behavior of customers and servers would be the $M_{n} / M_{n} / c / K+M_{n}$ queue which we analyze in Section 2 . This system receives customers according to a Poisson process with state-dependent arrival rates. Each server can change the service rate depending on the total number of customers in the system, namely, the system size. Similarly, each queued customer can update its abandonment rate with the changes in the system size. We set a limit $K$ on the maximum system size. And we assume that the underlying customer arrival, service and abandonment processes are independent of each other. In Section 2, we first obtain the steady-state system size distribution which is easily carried out by a birth-death modeling approach. Then, we focus on obtaining the queueing time distribution and its moments. To do this, we construct two continuous-time Markov chains (CTMC) one for an arbitrary customer and the other one for a customer that is eventually served. Both CTMC's are two-dimensional and from the moment an arbitrary (an eventually served) customer enters the queue the relevant CTMC traces how her position in the queue together with the system size can change until either the customer reaches a server or abandons from the queue (the customer is picked by a server). Thus, we assume that once service starts, the customers do not renege although they may experience varying service rates until they eventually leave the system upon completion of their service. We observe that these durations modeled via these CTMC's have Phase-type distributions (PTD). 
After characterizing the PTD's, we eventually obtain the Laplace transforms and moments of the queueing time random variables.

Such a flexible model is useful for researchers and practitioners in testing the results of the behavioral changes of the servers and customers they hypothesize as probable consequences of policy changes envisioned in delay systems. Obviously, assigning state-dependent rates at the individual level for each server and customer may not be always straightforward. For example, as pointed out by Akşin et al. (2016), estimating the state-dependent patience statistics of the queued customers from collected observations requires great care. However, with further technological advancements for handling big data, required estimates could be obtained with ease and the potential of employing the model developed here for new system designs can increase. One can use similar ideas to employ state-dependent Poisson processes instead of stationary Poisson demand processes that are traditionally assumed in models on maintenance (e.g., Katehakis and Derman, 1989, Katehakis and Melolidakis, 1995) and optimal replenishment (e.g., Katehakis and Smit, 2012).

Another potential area in which the $M_{n} / M_{n} / c / K+M_{n}$ queue can be employed is the approximate analysis of the $M_{n} / G I / c / K+G I$ queue, which we consider in Section 3. In the latter, queued customers have non-exponential patience times and service times also follow general distributions. For such systems, an exact analysis is not possible. The idea of employing a Markovian queue to approximately capture the performance measures of a multiserver queue with general service times is not new. For instance, for the $M / G I / c / K+G I$ system, Whitt (2005) suggests replacing the general service time distribution by an exponential distribution that has the same mean as the original service times. There are theoretical studies analyzing variants of the multi-server queues with general time-to-abandon distributions and exponential service times such as Baccelli and Hebuterne (1981) on the $M / M / c+G I$ queue, Movaghar (1998) on the $M_{n} / M / c+G I$ queue and Brandt and Brandt (1999) on the $M_{n} / M_{n} / c+G I$ queue (with the restriction that the total service rate is constant when all servers are busy). Computational difficulties arising in implementing these models can be overcome by considering state-dependent reneging rates. For instance, after suggesting that the $M / M / c / K+G I$ queue can approximate the $M / G I / c / K+G I$ queue, Whitt (2005) designs a method to obtain state-dependent reneging rates based on the original time-to-abandon distribution, which we adapt to our setting in Section 3. Thus, he constructs an $M / M / c / K+M_{n}$ queue for which he develops an approximate model to compute the queueing time distribution. Via this approximation, he eventually predicts the behavior of the original $M / G I / c / K+G I$ queue. Similarly, Brandt and Brandt (2002) suggest employing the $M_{n} / M_{n} / c+M_{n}$ queue to capture the performance of the $M_{n} / M_{n} / c+G I$ queue. They design an alternative method to set the statedependent reneging rates. Since their method depends on assuming that the total service rate must be a constant when all servers are busy, in Section 3, we have preferred adapting Whitt's method. Brandt and Brandt (2002) also state that the impact of the general service times may be reflected in the approxi- 
mating model if state-dependent service rates can be estimated based on the original service time distribution. This is still an open research question. For systems with a large number of servers, considering exponential distributions with the same mean as that of the original service times in all states appears to be providing sufficiently accurate estimates. For systems with few servers, another alternative - instead of fitting state-dependent service rates - could be employing the idea of scaling a single server $M_{n} / G I / 1 / K+G I$ queue. However, an exact analysis to obtain the queueing time and system size distributions in this queue is not yet available. If this analysis can be done, a single server $c$ times faster than one of the servers in the $M_{n} / G I / c / K+G I$ queue can provide accurate approximations for the latter system. We refer to Iravani and Balcıog̃lu (2008) who employ the scaling idea to approximately capture the performance measures of the $M / G I / c / K+G I$ queue.

The paper is organized as follows. In Section 2, we conduct the exact analysis of the $M_{n} / M_{n} / c / K+M_{n}$ queue. In Section 3, we adapt Whitt's (2005) method of fitting state-dependent reneging rates from the original timeto-abandonment distribution and then test the accuracy of the approximation we propose for predicting the behavior of the $M_{n} / G I / c / K+G I$ queue. We also observe that for systems with small number of servers, the prediction accuracy can improve if the characteristics of the original general service time can be somehow reflected into the $M_{n} / M_{n} / c / K+M_{n}$ queue. Section 4 presents conclusions and possible directions for future research.

\section{The exact analysis of the $M_{n} / M_{n} / c / K+M_{n}$ queueing system}

In this section, we analyze the $M_{n} / M_{n} / c / K+M_{n}$ queueing system to obtain its frequently employed steady-state performance measures. In this system, customers arrive according to a Poisson process with rate $\lambda_{n}$ when there are $n$ customers in the system (i.e., when the number of those receiving service and those waiting in the queue equals $n$ ) with $\lambda_{K}=0$. Each customer that has to wait in the queue has a random time-to-abandon and if their queueing time exceeds this random variable (r.v.), they renege and leave the system without receiving service. "Successful" customers are those who reach one of the $c$ parallel and identical servers before their time-to-abandon expires. Once their service starts, customers stay in the system until their service finishes. Service time and time-to-abandon r.v.s are exponentially distributed. We furthermore assume that $\mu_{n}$ and $\delta_{n-c}$, which are the total service and time-to-abandon rates, respectively, also depend on $n$. More specifically, we can consider

$$
\begin{aligned}
& \mu_{n}=\left\{\begin{array}{l}
\sum_{i=1}^{n} \mu_{n, i}, 1 \leq n \leq c, \\
\sum_{i=1}^{c} \mu_{n, i}, c+1 \leq n \leq K,
\end{array}\right. \\
& \delta_{k}=\sum_{i=1}^{k} \delta_{k, i}, \quad 1 \leq k=n-c \leq K-c,
\end{aligned}
$$

where $\mu_{n, i}\left(\delta_{k, i}\right)$ is the service (reneging/abandonment) rate of the server working on the $i$ th oldest customer taken into service (the $i$ th customer in the 
queue) when there are $n(k=n-c)$ customers in the system (queue). We can give the following example for further clarification: Suppose that customer A is the only queued customer and its time-to-abandon runs out at a rate of $\delta_{1,1}=$ $5\left(=\delta_{1}\right)$ and a second customer B arrives. With the new arrival, customers A and $\mathrm{B}$ take on abandonment rates of $\delta_{2,1}=2 / \delta_{2,2}=8$, respectively (with $\delta_{2}=$ $\delta_{2,1}+\delta_{2,2}$ ). If the next event is a service completion or the reneging of one of them from the queue, the remaining customer again assumes the abandonment rate of $\delta_{1,1}=5$. Thus, with information updates on the system size upon departures (either in the form of a service completion or abandonment) or a new customer arrival, each server and queued customer may change its service or abandonment rate, respectively.

Let $N(t)$ denote the number of customers in the system (the system state) at time $t$ which is a birth-death process with birth rate $\lambda_{n}$ in state $n$. The death rate $r_{n}$ in state $n$ is simply the total service rate when all the servers are not busy, and the sum of the total service and abandonment rates when there is at least one customer waiting in the queue:

$$
r_{n}=\left\{\begin{array}{c}
\mu_{n}, 1 \leq n \leq c \\
\mu_{n}+\delta_{n-c}, c+1 \leq n \leq K .
\end{array}\right.
$$

Let

$$
\pi_{n}=P(N=n)=\lim _{t \rightarrow \infty} P(N(t)=n \mid N(0)=i),
$$

for which there is always a unique solution due to the existence of abandonments and the system size limit $K$. These steady-state probabilities are obtained from the local balance equations

$$
\pi_{n} \lambda_{n}=\pi_{n+1} r_{n+1}, 0 \leq n \leq K-1,
$$

as

$$
\pi_{n}=\frac{\lambda_{n-1} \lambda_{n-2} \ldots \lambda_{0}}{r_{1} r_{2} \ldots r_{n}} \pi_{0}, 1 \leq n \leq K,
$$

where

$$
\pi_{0}=\left(1+\sum_{n=1}^{K} \frac{\lambda_{n-1} \lambda_{n-2} \ldots \lambda_{0}}{r_{1} r_{2} \ldots r_{n}}\right)^{-1} .
$$

Let $S$ be the event that a newly arriving customer eventually receives service and $A$ the event that she eventually abandons. Let also $W_{S}$ denote the queueing time r.v. for a successful customer. Then, (see, e.g., Brandt and Brandt, 2002) the probability that a customer eventually abandons is

$$
P_{A}=\frac{\sum_{n=c+1}^{K} \delta_{n-c} \pi_{n}}{\sum_{n=0}^{K} \lambda_{n} \pi_{n}},
$$

and the probability that a successful customer does not wait in the queue is

$$
P\left(W_{S}=0\right)=\frac{\sum_{n=0}^{c-1} \lambda_{n} \pi_{n}}{\left(1-P_{A}\right) \sum_{n=0}^{K} \lambda_{n} \pi_{n}} .
$$


To characterize the distribution and moments of the queueing time, in addition to $W_{S}$, we introduce $W_{T}$ and $W_{A}$ to denote the queueing time r.v. of all (total) and abandoning customers, respectively. Among these three r.v.s, one has the following relationships for their complementary queueing time distribution and their $k$ th moments, respectively:

$$
\begin{aligned}
P\left(W_{T} \geq w\right) & =\left(1-P_{A}\right) P\left(W_{S} \geq w\right)+P_{A} P\left(W_{A} \geq w\right), \\
E\left[W_{T}^{k}\right] & =\left(1-P_{A}\right) E\left[W_{S}^{k}\right]+P_{A} E\left[W_{A}^{k}\right] .
\end{aligned}
$$

We first obtain the statistics of $W_{T}$ for which we employ the r.v. $N^{\alpha}$ denoting the number of all customers found by a customer upon arrival with the following steady-state distribution:

$$
P\left(N^{\alpha}=n\right)=\frac{\lambda_{n} \pi_{n}}{\sum_{n=0}^{K} \lambda_{n} \pi_{n}} .
$$

Using $N^{\alpha}$, we can write

$$
\begin{aligned}
P\left(W_{T} \geq w\right) & =\sum_{n=c}^{K} P\left(W_{T} \geq w \mid N^{\alpha}=n\right) \frac{\lambda_{n} \pi_{n}}{\sum_{n=0}^{K} \lambda_{n} \pi_{n}}, \\
E\left[W_{T}^{k}\right] & =\sum_{n=c}^{K} E\left[W_{T}^{k} \mid N^{\alpha}=n\right] \frac{\lambda_{n} \pi_{n}}{\sum_{n=0}^{K} \lambda_{n} \pi_{n}} .
\end{aligned}
$$

Consider $W_{T}^{n-c+1}$, which is the queueing time r.v. of a "tagged" customer joining the queue when there are $n-c \geq 0$ customers in the queue (in other words, $\left.W_{T}^{n-c+1} \equiv W_{T} \mid N^{\alpha}=n\right)$. This r.v. is the time spent until absorption in a two-dimensional CTMC where the state $(i, j)$ denotes that the tagged customer is the $i$ th queued customer from the head of the queue when there are $j$ customers in the system. Thus, absorption occurs if this customer reneges or eventually reaches one of the $c$ parallel servers, the latter represented by a state of the form $(0, j), c \leq j$.

With $K$ as the limit on the system size, there are $K-n$ transient states, $(n-c+1, n+1),(n-c+1, n+2), \ldots,(n-c+1, K)$, that this CTMC can visit until the tagged customer either reneges or moves to a state $(n-c, j)$, $c \leq j<K$, i.e., making the tagged customer move one step closer to the head of the queue (or she is picked by one of the servers if $n=c$ ). In the same way, while in the transient state $(i, j), i>c$, this CTMC can visit $K-i-c+1$ transient states, $(i, j),(i, j+1), \ldots,(i, K)$ until the tagged customer either abandons or one more customer in front of it leaves the system either because of a service completion or abandonment.

Thus, starting from the state $(n-c+1, n+1)$, there are a total of $L=\sum_{i=1}^{n-c+1}(K-i-c+1)$ transient states that this CTMC can visit until absorption. Consequently, the r.v. $W_{T}^{n+1-c}$ follows a PTD. To characterize the underlying PTD (see, e.g. Altık, 1997, Section 2.8), we need the $L \times L$ matrix $\mathbf{T}$ that lists the transition rates among the transient states. Observe that from the transient state $(i, j)$ with $i>1, j>c$, this process can move 
to three neighboring transient states: with rate $\mu_{j}+\sum_{l=1}^{i-1} \delta_{j-c, l}$ to the state $(i-1, j-1)$, with $\lambda_{j}$ to the state $(i, j+1)$, and finally with rate $\sum_{l=i+1}^{j-c} \delta_{j-c, l}$ to the state $(i, j-1)$. Obviously the rate of leaving the state $(i, j)$ is also needed in $\mathbf{T}$ and is equal to $\mu_{j}+\lambda_{j}+\delta_{j-c}$.

Observe that with probability $1, W_{T}^{n+1-c}$ starts in the transient state $(n-$ $c+1, n+1)$, thus, the $1 \times L$ initial probability vector $\boldsymbol{\alpha}$ has 1 for the entry corresponding to this state and 0's otherwise. With the $L \times L$ identity matrix I, and the $L \times 1$ vector 1 of 1 's, for $W_{T}^{n-c+1}$, we have the moments

$$
E\left[\left(W_{T}^{n-c+1}\right)^{k}\right]=E\left[W_{T}^{k} \mid N^{\alpha}=n\right]=(-1)^{k} k ! \boldsymbol{\alpha} \mathbf{T}^{-n} \mathbf{1},
$$

and the Laplace transform (LT) for $W_{T}^{n-c+1}$

$$
\widetilde{f}_{T}^{n+1-c}(s)=\boldsymbol{\alpha}(s \mathbf{I}-\mathbf{T})^{-1} \mathbf{T}^{\mathbf{0}},
$$

where $\mathbf{T}^{\mathbf{0}}=-\mathbf{T} \mathbf{1}$. With these and noting that $P\left(W_{T}^{n-c+1} \geq w\right)=P\left(W_{T} \geq\right.$ $\left.w \mid N^{\alpha}=n\right)$ is obtained by numerically inverting the LT $\left(1-\widetilde{f}_{T}^{n+1-c}(s)\right) / s$, via Eq. (6), we can obtain $P\left(W_{T} \geq w\right)$ and $E\left[W_{T}^{k}\right]$.

When it comes to $W_{S}$, i.e., the queueing time of a successful customer, we can write

$$
P\left(W_{S} \geq w\right)=\sum_{n=c}^{K} P\left(W_{S} \geq w \mid N^{\alpha}=n, S\right) P\left(N^{\alpha}=n \mid S\right),
$$

where

$$
P\left(N^{\alpha}=n \mid S\right)=\frac{P\left(N^{\alpha}=n, S\right)}{1-P_{A}}=\frac{P\left(S \mid N^{\alpha}=n\right) P\left(N^{\alpha}=n\right)}{1-P_{A}},
$$

for which we need to compute $P\left(S \mid N^{\alpha}=n\right)$. Let $q_{i, j}$ denote the probability that the $i$ th customer from the head of the queue when there are a total of $j$ customers in the system will eventually be served. Then, with $q_{0, j}=1$, and considering the possible one-step transitions for the $i$ th customer in the queue in state $j$, we write

$$
\begin{aligned}
P\left(S \mid N^{\alpha}=n\right)= & q_{n-c+1, n+1}, \\
q_{i, j}= & \frac{\mu_{j}+\sum_{l=1}^{i-1} \delta_{j-c, l}}{\mu_{j}+\delta_{j-c}+\lambda_{j}} q_{i-1, j-1}+\frac{\lambda_{j}}{\mu_{j}+\delta_{j-c}+\lambda_{j}} q_{i, j+1} \\
& +\frac{\sum_{l=i+1}^{j-c} \delta_{j-c, l}}{\mu_{j}+\delta_{j-c}+\lambda_{j}} q_{i, j-1}, \quad i \geq 1, j \geq c .
\end{aligned}
$$

Combining all these we have

$$
P\left(N^{\alpha}=n \mid S\right)=\frac{\lambda_{n} \pi_{n} q_{n-c+1, n+1}}{\left(1-P_{A}\right) \sum_{n=0}^{K} \lambda_{n} \pi_{n}} .
$$

Now let us consider $W_{S}^{n-c+1}$, which is the queueing time r.v. of a tagged "successful" customer joining the queue when there are $n-c \geq 0$ customers 
in the queue. This r.v. is the time until absorption in one of the states $(0, j)$, $c \leq j$ (in which the customer reaches a server) of a two-dimensional CTMC where the state $(i, j)$ denotes that the tagged successful customer is the $i$ th queued customer from the head of the queue when there are $j$ customers in the system. And, $W_{S}^{n-c+1}$ also follows a PTD. Observe that the only difference we have here, compared to the PTD of $W_{T}^{n-c+1}$, is the lack of the possibility of abandonments as absorbing sates. This difference causes a change only in the transition rates in the $L \times L$ matrix $\mathbf{S}$ (in comparison to the matrix $\mathbf{T}$ introduced earlier) from the state $(i, j)$ with $i>1, j>c$ to the state $(i-1, j-1)$ as $\mu_{j}+\sum_{l=1}^{i} \delta_{j-c, l}$ (instead of $\mu_{j}+\sum_{l=1}^{i-1} \delta_{j-c, l}$ as in the matrix $\mathbf{T}$ ). Because for this tagged successful number, the exponential time for a customer in front of it to leave the system (with rate $\mu_{j}+\sum_{l=1}^{i-1} \delta_{j-c, l}$ ) has to be shorter than its own exponential time-to-abandonment (with rate $\delta_{j-c, i}$ ).

Then, with $\mathbf{S}$ instead of $\mathbf{T}$ in Eq. (7), we can obtain $E\left[\left(W_{S}^{n-c+1}\right)^{k}\right] \equiv$ $E\left[W_{S}^{k} \mid N^{\alpha}=n, S\right]$ to compute the $k$ th moment and variance of a successful customer's queueing time as follows:

$$
\begin{aligned}
E\left[W_{S}^{k}\right] & =\sum_{n=c}^{K} E\left[W_{S}^{k} \mid N^{\alpha}=n, S\right] \frac{\lambda_{n} \pi_{n} q_{n-c+1, n+1}}{\left(1-P_{A}\right) \sum_{n=0}^{K} \lambda_{n} \pi_{n}}, \\
\operatorname{Var}\left[W_{S}\right] & =E\left[W_{S}^{2}\right]-E\left[W_{S}\right]^{2} .
\end{aligned}
$$

Similarly, $\mathbf{S}$ instead of $\mathbf{T}$ in Eq. (8) would give the LT for $W_{S}^{n-c+1}$, which when numerically inverted, yields $P\left(W_{S}^{n-c+1} \geq w\right) \equiv P\left(W_{S} \geq w \mid N^{\alpha}=n, S\right)$, and via Eqs. (9) and (11), the probability that a successful customer's queueing time does not exceed $w$ is given by

$$
P\left(W_{S} \geq w\right)=\sum_{n=c}^{K} P\left(W_{S} \geq w \mid N^{\alpha}=n, S\right) \frac{\lambda_{n} \pi_{n} q_{n-c+1, n+1}}{\left(1-P_{A}\right) \sum_{n=0}^{K} \lambda_{n} \pi_{n}} .
$$

With the distributions and the moments of the queueing time r.v.s $W_{T}$ and $W_{S}$ in hand, those of $W_{A}$ can be found from Eq. (5).

\section{An application: Approximating the $M_{n} / G I / c / K+G I$ queue}

In this section, we develop an approximation method that employs the $M_{n} / M$ $/ c / K+M_{n}$ queue and test its accuracy in capturing the steady-state performance measures of the $M_{n} / G I / c / K+G I$ queueing system. In the latter, customers arrive according to a Poisson process with state-dependent rate $\lambda_{n}$ and the independent and identically distributed (i.i.d.) service time $(B)$ and time-to-abandon $(R)$ r.v.s follow general distributions. The idea for approximation is to construct an $M_{n} / M / c / K+M_{n}$ queue with the same arrival process as in the original system and with exponentially distributed service times having a rate of $\mu=1 / E[B]$ that would capture the behavior of the original queueing system. For examples involving a small number of servers, we 
also demonstrate how state-dependent service rates can improve the accuracy of the approximation.

To construct the approximating $M_{n} / M / c / K+M_{n}$ queue, we extend the idea proposed by Whitt (2005) to set the state-dependent abandonment rates based on the original time-to-abandon distribution. Let

$$
h_{R}(t)=\frac{f_{R}(t)}{\bar{F}_{R}(t)},
$$

be the hazard rate function of the time-to-abandon r.v. $R$ where $f_{R}()$ and $\bar{F}_{R}()$ stand for the density and cumulative distribution functions, respectively. Suppose that at an observation instant, we see $k$ customers in the queue. If one can find how long each of these customers have been in the queue, for each one the hazard rate value can be computed and can be substituted for the state-dependent abandonment rates in the approximating $M_{n} / M / c / K+M_{n}$ queue. Since this is not possible, similar to Whitt, we simply assume that the $j$ th customer from the head of the queue must have waited, on average, $1 / \lambda_{c+j}$ time units longer than the $j+1$ st customer from the head of the queue, ignoring that additional customers may have joined and left between the arrival instants of the $j$ th and $j+1$ st customers. With the same reasoning, since the last customer joined the queue, the mean length of time expended until our observation instant is assumed to be the mean backward sojourn time in the interval from the arrival of the $k$ th until that of the $k+1$ st customer, which is equal to $1 / \lambda_{c+k}$. Then, we can set the following

$$
\delta_{j, k}=h_{R}\left(\sum_{i=j}^{k} \frac{1}{\lambda_{c+i}}\right), \quad 1 \leq j \leq k, \quad 1 \leq k \leq K-c-1 .
$$

If $k=K-c$, since $\lambda_{K}=0$ we cannot think that we are in the middle of the time interval until the arrival of the next customer. Instead of this, we can think that we are in the middle of the interval until the next service completion (ignoring probable abandonments again) and use the mean backward recurrence time of such an interval, namely $1 / c \mu$, for the expected length of the time the last customer has been in the system and write

$$
\delta_{j, K-c}=h_{R}\left(\sum_{i=j}^{K-c-1} \frac{1}{\lambda_{c+i}}+\frac{1}{c \mu}\right), \quad 1 \leq j \leq K-c .
$$

Eventually Eq. (2) provides the state-dependent abandonment rates $\delta_{k}$ 's.

We have not been successful with a similar approach to set $\mu_{n, i}$ 's then to obtain $\mu_{n}$ 's, and this remains as an open research question. As demonstrated in the numerical examples to follow, most of the time, especially when $c$ is large, setting $\mu_{n}=1 / E[B]$ for $n=1, \ldots, K$ and using $\delta_{k}$ 's determined as described above in the $M_{n} / M / c / K+M_{n}$ queue provides accurate predictions. Yet, to demonstrate the potential gains we can have with state-dependent service rates, especially when $c$ is small, we have estimated $\hat{\mu}_{n}$ in the following 
way. From the simulation of the original $M_{n} / G I / c / K+G I$ queue, we have estimated $\hat{p}_{n, n-1}$, namely the probability of moving from state $n$ to $n-1$. Now consider the $M_{n} / M_{n} / c / K+M_{n}$ queue in which

$$
p_{n, n-1}= \begin{cases}\frac{\mu_{n}}{\mu_{n}+\lambda_{n}}, & n=1, \ldots, c \\ \frac{\mu_{n}+\delta_{n-c}}{\mu_{n}+\delta_{n-c}+\lambda_{n}}, & n=c+1, \ldots, K .\end{cases}
$$

With $\lambda_{n}$ in hand and $\delta_{n-c}$ set as explained above, we substitute $\hat{p}_{n, n-1}$ for $p_{n, n-1}$ above and solve for $\hat{\mu}_{n}$. In a way, given $\delta_{n-c}$ estimates, this is the best $\mu_{n}$ estimates one can hope to obtain from an algorithm to be worked on in the future. With these rates in hand, one can obtain the statistics of the $M_{n} / M_{n} / c / K+M_{n}$ queue that serve as approximate predictions for the $M_{n} / G I / c / K+G I$ queue.

In order to assess the accuracy of the proposed approximations, we have considered examples with different service and time-to-abandon distributions, different state-dependent arrival rate functions, etc. Table 1 is inspired by the small size call center system with five agents studied in Akşin et al. (2016) where no more than $(K=) 18$ customers were observed in the system at any time. We assumed a single type of customer class as against their two-priority classes of customers and fit the state-dependent Poisson arrival rates as

$$
\lambda_{n}= \begin{cases}2, & n=0, \ldots, 5, \\ 2\left(1-\left(\frac{8(n-5)^{4}}{100,000}-\frac{29(n-5)^{3}}{10,000}+\frac{32(n-5)^{2}}{1,000}-\frac{27(n-5)}{1,000}+0.0505\right)\right), & n=6, \ldots, 17 .\end{cases}
$$

by treating the function given in their Figure 6 (for high-priority class) as the probability of a customer balking/hanging up when finding all servers busy. Instead of exponential service and time-to-abandonment distributions considered by Akşin et al., we assume that the service times follow a $\mathrm{H}_{2}$ (2phase Hyperexponential, $\left.H 2\left(a, \omega_{1}, \omega_{2},\right)\right)$ distribution with density

$$
b(x)=a \omega_{1} e^{-\omega_{1} x}+(1-a) \omega_{2} e^{-\omega_{2} x},
$$

and with $E[B]=2$ and $c_{B}^{2}=5$ (variance-to-squared mean ratio), and the time-to-abandon distribution is 8-stage Erlang $(\operatorname{Erlang}(\mu, k=8))$ with density

$$
b(x)=\frac{\mu(\mu x)^{k-1} e^{-\mu x}}{(k-1) !}
$$

and with $E[R]=4$ and $c_{R}^{2}=0.125$. Thus, compared to the highly variable service times, time-to-abandon r.v. is longer and reveals very little variation.

In all tables presenting our numerical study, the reference values for the performance measures listed in column 1 are obtained by simulating the original $M_{n} / G I / c / K+G I$ queue and are provided in column 2. With a warm-up period long enough to have 10,000 arrivals, 10 replications each of which is long enough to have one million arrivals have yielded close to 0 half-widths for the $95 \%$ confidence interval, thus, we omit to show them in this column. In the third column, we list the estimates of the $M_{n} / M / c / K+M_{n}$ queue approximation for the listed performance measures. The last column (in Tables 1 
Table 1 Approximations for the $M / H 2(0.689,10,0.161) / 5 / 18+\operatorname{Er}(8,2)$ queue with $\lambda_{0}=2$

\begin{tabular}{cccc}
\hline $\begin{array}{c}\text { Performance } \\
\text { Measure }\end{array}$ & $\begin{array}{c}M_{n} / G I / c / K+G I \\
\text { Simulation }\end{array}$ & $\begin{array}{c}M_{n} / M / c / K+M_{n} \\
\text { Approximation }\end{array}$ & $\begin{array}{c}M_{n} / M_{n} / c / K+M_{n} \\
\text { Approximation }\end{array}$ \\
\hline$P_{A}$ & 0.04 & 0.02 & $\mathbf{0 . 0 4}$ \\
$P\left(W_{S}=0\right)$ & 0.71 & 0.61 & $\mathbf{0 . 7 2}$ \\
$P\left(W_{S} \geq 0.1\right)$ & 0.26 & 0.36 & $\mathbf{0 . 2 6}$ \\
$P\left(W_{S} \geq 0.6\right)$ & 0.19 & 0.22 & $\mathbf{0 . 1 9}$ \\
$P\left(W_{S} \geq 1\right)$ & 0.15 & 0.14 & 0.14 \\
$E\left[W_{S}\right]$ & 0.38 & 0.33 & $\mathbf{0 . 3 6}$ \\
$\operatorname{Var}\left[W_{S}\right]$ & 0.72 & 0.35 & $\mathbf{0 . 6 6}$ \\
\hline
\end{tabular}

and 2) is for the corresponding values estimated from the $M_{n} / M_{n} / c / K+M_{n}$ queue with $\hat{\mu}_{n}$ obtained as described above. In both approximations (one with $\mu=1 / E[B]$ and the other with $\left.\hat{\mu}_{n}\right)$, the analysis in Section 2 is employed. In specific, for $P\left(W_{S} \geq w\right)$ in Eq. (14), the LT's of $W_{S}^{n-c+1}$ are numerically inverted by the fixed Talbot algorithm in Abate and Valkó (2014). The other performance measures $P_{A}, P\left(W_{S}=0\right), E\left[W_{S}\right]$, and $\operatorname{Var}\left[W_{S}\right]$ are obtained from Eqs. (3), (4), (12), and (13), respectively.

Table 1 presents an example in which most of the successful customers (71\%) find idle servers and among those who wait in the queue, a mere $4 \%$ abandons out of impatience. Thus, this is not a system operating under heavy customer traffic. In the last two columns, the bold-faced figures are those approximations closer to the corresponding reference value. The estimates in the last column are quite accurate. Thus, state-dependent service rates that would capture the characteristics of general service times can enhance the quality of the approximation significantly for delay systems with a small number of servers.

Table 2 is inspired from the medium size (regarding the number of servers) numerical examples of Iravani and Balcıoglu (2008). Here both the service and time-to-abandon r.v.s are assumed to be lognormal with $E[B]=1=1 / \mu$ and $c^{2}=4$ (which is also equal to the variance $\sigma^{2}$ ). The $\operatorname{lognormal}\left(E[B], \sigma^{2}\right)$ density function is

$$
b(x)=\frac{e^{-\frac{(\ln x-p)^{2}}{2 q^{2}}}}{x q \sqrt{2 \pi}},
$$

where

$$
p=\ln \left(\frac{E[B]}{\sqrt{1+\sigma^{2} / E[B]^{2}}}\right), q=\sqrt{\ln \left(1+\sigma^{2} / E[B]^{2}\right)} .
$$

This is an example in which the patience times of the queued customers are also as variable as the service times. We consider a linear function for setting the state-dependent Poisson arrival rates as

$$
\lambda_{n}= \begin{cases}24, & n=0, \ldots, 20 \\ 24(1-(n-20)) / 20, & n=20, \ldots, 40 .\end{cases}
$$


Table 2 Approximations for the $M / L N(1,4) / 20 / 40+L N(1,4)$ queue with $\lambda_{0}=24$

\begin{tabular}{cccc}
\hline $\begin{array}{c}\text { Performance } \\
\text { Measure }\end{array}$ & $\begin{array}{c}M_{n} / G I / c / K+G I \\
\text { Simulation }\end{array}$ & $\begin{array}{c}M_{n} / M / c / K+M_{n} \\
\text { Approximation }\end{array}$ & $\begin{array}{c}M_{n} / M_{n} / c / K+M_{n} \\
\text { Approximation }\end{array}$ \\
\hline$P_{A}$ & 0.12 & 0.13 & 0.15 \\
$P\left(W_{S}=0\right)$ & 0.41 & $\mathbf{0 . 4 1}$ & 0.42 \\
$P\left(W_{S} \geq 0.1\right)$ & 0.33 & 0.31 & $\mathbf{0 . 3 3}$ \\
$P\left(W_{S} \geq 0.2\right)$ & 0.14 & 0.12 & $\mathbf{0 . 1 5}$ \\
$P\left(W_{S} \geq 0.3\right)$ & 0.05 & 0.04 & 0.06 \\
$E\left[W_{S}\right]$ & 0.08 & 0.08 & 0.08 \\
$\operatorname{Var}\left[W_{S}\right]$ & 0.01 & 0.01 & 0.01 \\
\hline
\end{tabular}

Table 2 presents an example in which less than half of the successful customers (41\%) find idle servers and among those who wait in the queue, a relatively high $(12 \%)$ rate of abandonment is observed. Thus, this is a highly busy system. And we see that both approximations are comparable to one another and simply assuming $\mu=1 / E[B]$ for all states yields highly accurate approximations.

In Table 3, we have a larger system with 100 servers where service times, with $E[B]=2$ and $c_{B}^{2}=2$, follow an $\mathrm{H} 2(0.3565,10,0.3275)$ distribution and time-to-abandon distribution is $\operatorname{Er}(2,2)$ with $E[R]=1$ and $c_{R}^{2}=0.5$. Thus, compared to service times, customer patience times tend to be shorter and less variable. We consider a convex function for setting the state-dependent Poisson arrival rates as

$$
\lambda_{n}= \begin{cases}60, & n=0, \ldots, 100, \\ 60\left(1-((n-100) / 40)^{1 / 4}\right), & n=100, \ldots, 140 .\end{cases}
$$

This function captures the behavior of customers who are sensitive to the delay which reduces $\lambda_{n}$ sharply with increase in $n$. Consequently, this is an example in which most of the successful customers (63\%) find idle servers and among those who wait in the queue almost no one $(0.002 \%)$ abandons. In this regard, similar to the case in Table 1, we again have a queueing system operating under light conditions. The basic difference is that this is a much larger system in terms of the number of servers. Since it was not practical, we did not estimate $\hat{\mu}_{n}$ from the simulation of the $M / H 2(0.3565,10,0.3275) / 100 / 140+$ $\operatorname{Er}(2,2)$ queue, thus, we do not present estimates of the $M_{n} / M_{n} / c / K+M_{n}$ queue approximation. However, there is a hardly a need for this since the $M_{n} / M / c / K+M_{n}$ queue approximation with $\mu=1 / E[B]$ for all states yields highly accurate approximations.

Table 4 is inspired by an example in Whitt (2005) where (as in Table 2) both the service and time-to-abandon r.v.s are assumed to be lognormal with $E[B]=1$ and $c^{2}=4$ (which is also the variance). We consider a concave function for setting the state-dependent Poisson arrival rates as

$$
\lambda_{n}= \begin{cases}102, & n=0, \ldots, 100, \\ 102\left(1-((n-100) / 40)^{4}\right), & n=100, \ldots, 140 .\end{cases}
$$


Table 3 Approximations for the $M / H 2(0.3565,10,0.3275) / 100 / 140+E r(2,2)$ queue with $\lambda_{0}=60$

\begin{tabular}{ccc}
\hline $\begin{array}{c}\text { Performance } \\
\text { Measure }\end{array}$ & $\begin{array}{c}M_{n} / G I / c / K+G I \\
\text { Simulation }\end{array}$ & $\begin{array}{c}M_{n} / M / c / K+M_{n} \\
\text { Approximation }\end{array}$ \\
\hline$P_{A}$ & 0.002 & 0.004 \\
$P\left(W_{S}=0\right)$ & 0.63 & 0.61 \\
$P\left(W_{S} \geq 0.01\right)$ & 0.31 & 0.32 \\
$P\left(W_{S} \geq 0.05\right)$ & 0.15 & 0.13 \\
$P\left(W_{S} \geq 0.1\right)$ & 0.06 & 0.04 \\
$E\left[W_{S}\right]$ & 0.02 & 0.02 \\
$\operatorname{Var}\left[W_{S}\right]$ & 0.002 & 0.001 \\
\hline
\end{tabular}

Table 4 Approximations for the $M / L N(1,4) / 100 / 140+L N(1,4)$ queue with $\lambda_{0}=60$

\begin{tabular}{ccc}
\hline $\begin{array}{c}\text { Performance } \\
\text { Measure }\end{array}$ & $\begin{array}{c}M_{n} / G I / c / K+G I \\
\text { Simulation }\end{array}$ & $\begin{array}{c}M_{n} / M / c / K+M_{n} \\
\text { Approximation }\end{array}$ \\
\hline$P_{A}$ & 0.05 & 0.05 \\
$P\left(W_{S}=0\right)$ & 0.48 & 0.47 \\
$P\left(W_{S} \geq 0.1\right)$ & 0.15 & 0.14 \\
$E\left[W_{S}\right]$ & 0.04 & 0.04 \\
$\operatorname{Var}\left[W_{S}\right]$ & 0.003 & 0.003 \\
\hline
\end{tabular}

This function captures the behavior of customers who are relatively insensitive to the delay, thus, $\lambda_{n}$ does not decrease sharply with increase in $n$. Consequently, this is an example in which less than half of the successful customers $(48 \%)$ find idle servers and $5 \%$ of those who wait in the queue abandon. In this regard, similar to the case in Table 2, we again have a queueing system in which servers are busy most of the time. Since we did not estimate $\hat{\mu}_{n}$ from the simulation of the $M / L N(1,4) / 100 / 140+L N(1,4)$ queue, we do not present the estimates of $M_{n} / M_{n} / c / K+M_{n}$ queue approximation. The $M_{n} / M / c / K+M_{n}$ queue approximation with $\mu=1 / E[B]$ for all states yields highly accurate approximations.

Our numerical study indicates that for small size queueing systems under relatively light traffic, via state-dependent service rates that would capture the statistical characteristics of the general service time distributions, one can improve the accuracy of the Markovian approximation for the $M_{n} / G I / c / K+$ $G I$ queue. However, in general, simply choosing $\mu=1 / E[B]$ for all states and fitting abandonment rates based on the original general time-to-abandon distribution, one can obtain highly accurate estimates.

\section{Conclusion}

In this paper, we study the $M_{n} / M_{n} / c / K+M_{n}$ queue in which the rates of the exponential interarrival, service and reneging times can change with the change in system size. We allow that state-dependent service/abandonment rates can be defined for each server/queued customer. Obtaining the steady-state distribution of the system size in this setting is carried out by a birth-death 
process. For obtaining the queue time distribution, we consider CTMC's capturing how a customer's (both for an arbitrary customer and a successfully served customer) position in the queue together with the system size may change if she decides to join the queue. Observing that the absorption times in these CTMC's have PTD, we provide a numerical approach with which the queueing time distribution and their moments can be evaluated. With its flexibility stemming from the assumptions concerning state-dependent rates, the model can serve researchers and practitioners who would like to answer what-if questions on how the system performance may change when policy changes lead to some anticipated behavioral changes of customers and servers. As a secondary goal, we employ the developed model to approximately analyze the $M_{n} / G I / c / K+G I$ queue. Numerical examples provided attest to the high accuracy of the proposed approximation in predicting the performance measures of the $M_{n} / G I / c / K+G I$ queue.

Since a customer may experience varying service rates after she starts receiving service, one can explore a system in which reneging after service is also allowed. Similarly, call centers providing a call-back option or serving multiple priority classes can be analyzed by allowing state-dependent arrival, service, and reneging processes. Resulting models may later on be employed to approximately analyze the multi-class call centers providing a call-back option in which service times and times-to-abandonment follow general distributions. In this quest, the need for setting state-dependent service rates based on the original service times may become prevalent.

\section{Acknowledgements}

This work was supported in part by TÜBİTAK, The Scientific and Technological Research Council of Turkey, under the Grant No. 213M428. The authors thank the two anonymous referees and the editors for their invaluable suggestions to improve the manuscript.

\section{References}

Abate, J., and P.P. Valkó. "Multi-precision Laplace transform inversion", International Journal for Numerical Methods in Engineering, Vol. 60, No. 5, 979-993 (2014).

Akşin, Z., B. Ata, S. M. Emadi, C. Su. "Impact of delay announcements in call centers: An empirical approach", Operations Research, http://dx.doi.org/ 10.1287/opre.2016.1542 (2016).

Altıok, T. "Performance Analysis of Manufacturing Systems," Springer, New York (1997).

Armony, M., N. Shimkin, W. Whitt. "The impact of delay announcements in many-server queues with abandonment", Operations Research, Vol. 57,No. 1, 66-81 (2009). 
Armony, M., E. Plambeck, and S. Seshardi. "Sensitivity of optimal capacity to customer impatience in an unobservable M/M/S queue (Why you shouldnt shout at the DMV)", Manufacturing $\&$ Service Operations Management, Vol. 11, No. 1, 19-32 (2009).

Ata, B., and S. Shneorson. "Dynamic control of an M/M/1 service system with adjustable arrival and service rates", Management Science, Vol. 52, No. 11, 1778-1791 (2006).

Baccelli, F., and G. Hebuterne. "On queues with impatient customers", Performance '81, ed. F.J. Kylstra (North Holland, Amsterdam 1981), Vol. 32, 159-179 (1981).

Batt, R. J., and C. Terwiesch. "Early task initiation and other load-adaptive mechanisms in the emergency department", Operations Research, http://dx. doi.org/10.1287/mnsc.2016.2516 (2016).

Berry Jaeker, J. A., and A. L. Tucker. "Past the point of speeding up: The negative effects of workload saturation on efficiency and patient severity", Management Science, http://dx.doi.org/10.1287/mnsc.2015.2387 (2016).

Brandt A., and M. Brandt. "On the $M(n) / M(m) / s$ queues with impatient calls", Performance Evaluation, Vol. 35, Issue 1-2, 1-18 (1999).

Brandt A., and M. Brandt. "Asymptotic results and a Markovian approximation for the $M(n) / M(m) / S+G I$ system, Queueing Systems: Theory and Applications, Vol. 41, 73-94 (2002).

Iravani, F. and B. Balcıoglu. "Approximations for the $M / G I / N+G I$ type call center", Queueing Systems, Vol. 58, 137-153 (2008).

Jouini, O., Z. Akşin, and Y. Dallery. "Call centers with delay information: Models and insights", Manufacturing \& Service Operations Management,

Vol. 13, No. 4, 534-548 (2011).

Jouini, O., O. Z. Akşin, F. Karaesmen, M. S. Aguir, and Y. Dallery. "Call center delay announcement using a newsvendor-like performance criterion", Production and Operations Management, Vol. 24, No. 4, 587-604 (2015).

Elmaghraby, W. and P. Keskinocak. "Dynamic pricing in the presence of inventory considerations: Research overview, current practices, and future directions, Management Science, Vol. 49, No. 10, 12871309 (2003).

Katehakis M. N. and C. Derman. "On the maintenance of systems composed of highly reliable components", Management Science, Vol. 35, No. 5, 1628 (1989).

Katehakis M. N. and C. Melolidakis. "On the optimal maintenance of systems and control of arrivals in queues", Stochastic Analysis and Applications, Vol. 13, No. 2, 137-164 (1995).

Katehakis M.N and L.C. Smit. "Efficient Algorithms for Computing an Optimal (R,Q) Policy in Continuous Review Stochastic Inventory Systems with Quantity Discounts", Annals of Operations Research, Vol. 200, No. 1, 279-298 (2012).

Movaghar, A. "On queueing with customer impatience until the beginning of service", Queueing Systems, Vol. 29, 337-350 (1998).

Naor, P. "The regulation of queue size by levying tolls, Econometrica, Vol. 37, No. 1, 1524 (1969). 
Tan, T. F., and S. Netessine. "When does the devil make work? An empirical study of the impact of workload on worker productivity", Management Science, Vol. 60, No. 6, 15741593 (2014).

Whitt, W. "Engineering solution of a basic call center model", Management Science, Vol. 51, No. 2, 221-235 (2005). 